Revue

Revue de l'histoire des religions

del'histoire des religions

3 | 2016

Varia

\title{
Le bien et le mal dans les psychostasies égyptienne et chrétienne
}

Good and Evil in the Egyptian and Christian Psychostasias

\section{Hanane Gaber}

\section{OpenEdition \\ Journals}

Édition électronique

URL : http://journals.openedition.org/rhr/8568

DOI : $10.4000 /$ rhr.8568

ISSN : 2105-2573

\section{Éditeur}

Armand Colin

\section{Édition imprimée}

Date de publication : 1 septembre 2016

Pagination : 305-327

ISBN : 978-2-200-93061-5

ISSN : 0035-1423

Référence électronique

Hanane Gaber, "Le bien et le mal dans les psychostasies égyptienne et chrétienne », Revue de l'histoire des religions [En ligne], 3 | 2016, mis en ligne le 01 septembre 2019, consulté le 05 septembre 2020. URL : http://journals.openedition.org/rhr/8568 ; DOI : https://doi.org/10.4000/rhr.8568 


\section{Le bien et le mal dans les psychostasies égyptienne et chrétienne}

L'objet de cette étude est de classer les acteurs divins dans les psychostasies égyptienne et chrétienne, d'y examiner l'interaction des protagonistes et d'établir les liens des « dieux / génies / anges » avec le bien, ceux des "démons » avec le mal. Cette comparaison permet, par exemple, de déterminer le rôle des quarante-deux assesseurs et de la dévoreuse, souvent considérés comme figures démoniaques. Les quarante-deux dieux et le monstre dévoreur agissent en fait comme des bourreaux ou des génies exécuteurs, dont la fonction est analogue à celle des anges qui, au terme du Jugement dernier, punissent le diable et ses alliés. Quant à ces derniers, ils correspondent à Seth et à ses acolytes qui sont les vrais démons de la psychostasie égyptienne.

\section{Good and Evil in the Egyptian and Christian Psychostasias}

This study aims to classify the divine participants in the Egyptian and Christian psychostasias, to examine the interaction of its protagonists, to establish the connection between "gods / genies / angels" and good, and the connection of "demons" with evil. This comparison enables us to determine, for example, the function of the forty-two assessors and the devourer, which are often regarded as demonic figures. The forty-two gods and the monster in fact act as executioner genies, whose function is similar to the angels at the Last Judgement, when they punish the Devil and his associates. The latter are equivalent to Seth and his acolytes, who are the real demons in the Egyptian psychostasia. 
Une contribution au catalogue de l'exposition Dieux, génies et démons d'Égypte ${ }^{1}$, portant sur la classification des acteurs divins dans la psychostasie, m'a incitée à comparer cette scène avec son pendant chrétien du Jugement dernier afin d'évaluer les différences et les similitudes des fonctions de leurs intervenants. Dépassant largement le cadre de l'exposition, cette étude fait l'objet du présent article ${ }^{2}$.

La psychostasie consiste en la pesée du cœur ou de l'âme du défunt devant Osiris ou devant le Christ, procédure dont le résultat positif ou négatif détermine le sort réservé à la personne jugée dans l'au-delà. L'identification de certains acteurs de la psychostasie égyptienne ${ }^{3}$ en tant que «génie » ou "démon » n'est pas aisée. $\mathrm{Au}$ contraire du substantif et de l'adjectif « dieu, divin (nțr, $n \underline{t} r y) »$, ces deux termes, qui puisent leurs racines respectivement dans le latin genius $^{4}$ et le grec daimôn ${ }^{5}$, font défaut dans les textes

1. Arnaud Quertinmont (éd.), Dieux, génies et démons d'Égypte. À la rencontre d'Osiris, Anubis, Isis, Hathor, Rê et les autres... [Exposition du 21 mai au 20 novembre 2016 au Musée royal de Mariemont, Morlanwelz], Prais, 2016, p. 285-289.

2. Mes vifs remerciements à Françoise Labrique, l'higoumène Elias-Patrick Leroy, Jérôme Rizzo et Thierry Van Compernolle pour la lecture de cet article et leurs remarques avisées. Ma reconnaissance va également à Arnaud Delhove pour la discussion enrichissante que nous avons eue sur ce thème.

3. Joachim Spiegel, Die Idee des Totengerichts in der ägyptischen Religion, Leipziger Ägyptologische Studien 2, 1935 ; Jean Yoyotte, « Le jugement des morts dans l'Égypte ancienne », dans Le jugement des morts, Sources Orientales 4, Paris, 1961, p. 15-80 ; Reinhard Grieshammer, Das Jenseitsgericht in den Sargtexten, Ägyptologische Abhandlungen 20, 1970 ; Christine Seeber, Untersuchungen zur Darstellung des Totengerichts im Alten Ägypten, Münchner ägyptologische Studien 35, 1976 ; Jan Assmann, Maât. L'Égypte pharaonique et l'idée de justice sociale, [Paris], 1989, p. 70-84 ; John Gwyn Griffiths, The Divine Verdict : a Study of Divine Judgement in the Ancient Religions, Studies in the History of Religions 52, Leiden, 1991, p. 224-236; Miriam Lichtheim, Maat in Egyptian Autobiographies and Related Studies, Orbis biblicus et orientalis 120, 1992, p. 103-144 ; Jan Assmann, Mort et au-delà dans l'Égypte ancienne, Paris, 2003, p. 114-140 ; Hanane Gaber, « Deux variantes de la scène de la psychostasie (chapitres 30 et 125 du Livre des Morts) », Revue d'Égyptologie 60, 2009, p. 1-16; Hanane Gaber, «L'interaction des dieux, des génies et des démons dans la scène de la psychostasie », dans A. Quertinmont (éd.), op. cit., p. 285-289..

4. Le genius personnifiait la puissance d'un être ou d'une chose ou d'un lieu, telle qu'elle se formait au moment de sa naissance ou de sa constitution : John Scheid, La religion des Romains, Paris, 1998, p. 137.

5. Homère a recours à daimôn pour qualifier une puissance divine que l'on ne peut ou ne veut pas nommer, d'où le sens de divinité ou de destin : Pierre Chantraine, Dictionnaire étymologique de la langue grecque. Histoire des mots, 
égyptiens ${ }^{6}$. Par conséquent, leur usage dans la classification des êtres divins relève, en égyptologie, de la subjectivité ${ }^{7}$. Chacun de ces deux mots recouvre alors des significations variées. "Génie » est employé pour décrire les rôles spécifiques de quelques êtres ou leurs fonctions bénéfiques ${ }^{8}$. Certains égyptologues ont recours à daimôn pour désigner un être divin qui ne bénéficie pas d'un culte $^{9}$, alors que d'autres se réfèrent au démon judéo-chrétien pour qualifier les êtres divins maléfiques, perçus comme les représentants du $\operatorname{chaos}^{10}$.

Il s'agit donc d'examiner l'interaction des protagonistes dans les deux psychostasies et de définir les liens des « dieux / génies / anges » avec le bien, ceux des « démons » avec le mal. Dans la psychostasie

Paris, 1999. p. 246 ; selon Empédocle, daimôn désigne de façon indéterminée une divinité, masculine ou féminine, qu'elle soit identifiée ou non : Jean-François Balaudé, Le vocabulaire des Présocratiques, Paris, 2002, p. 15. Pour un exposé plus détaillé sur la signification et l'évolution de daimôn, voir Panagiotis Kousoulis, « The Demonic Lore of Ancient Egypt : Questions on Definitions », dans Panagiotis Kousoulis (éd.), Ancient Egyptian Demonology : Studies on the Boundaries between the Demonic and the Divine in Egyptian Magic, Orientalia Lovaniensia Analecta 175,2011 , p. X-XI.

6. Herman te Velde, « Dämonen », dans Wolfgang Helck, Eberhard Otto, Lexikon der Ägyptologie I, Wiesbaden, 1975, col. 980 ; Dimitri Meeks, « Demons », dans The Oxford Encyclopedia of Ancient Egypt I, Oxford, New York, 2001, p. 375 ; Rita Lucarelli, "Demons in the Book of the Dead», dans Burkhard Backes, Irmtraut Munro, Simone Stöhr (éd.), Totenbuch-Forschungen. Gesammelte Beiträge des 2. Internationalen Totenbuch-Symposiums 2005, Studien zum altägyptischen Totenbuch 11, 2006, p. 203, $\mathrm{n}^{\circ} 1$; Kasia Szpakowska, « Demons in Ancient Egypt», dans Religion Compass 3, 2009, p. 799 ; P. Kousoulis, op. cit., p. XI-XIV.

7. Dimitri Meeks, « Génies, anges, démons en Égypte », Sources orientales 8, 1971, p. 19-21 ; id., «Demons », op. cit., p. 375.

8. D. Meeks, «Génies, ... », op. cit., p. 18-84 ; D. Meeks, « Demons », op. cit., p. 375 ; R. Lucarelli, op. cit., p. 210 ; K. Szpakowska, op. cit., p. 799.

9. D. Meeks, «Génies,... », op. cit., p. 20 ; D. Meeks, «Demons », op. cit., p. 375 ; Rita Lucarelli, «Towards a Comparative Approach to Demonology in Antiquity: The Case of Ancient Egypt and Mesopotamia », Archiv für Religionsgeschichte 14, 1, 2013, p. 16, $\mathrm{n}^{\circ} 26$. Sur cette acception du terme daimôn : P. Chantraine, op. cit., p. 246.

10. R. Lucarelli, «Towards...», op. cit., p. 12 ; Rita Lucarelli, «Demons (Benevolent and Malevolent) », dans UCLA Encyclopedia of Egyptology, 2010, p. 2 : <http://escholarship.org/uc/item/1r72q9vv>; K. Szpakowska, op. cit., p. 799. Sur les classifications des catégories d'êtres repertoriées comme démons : R. Lucarelli, « Demons in the Book of the Dead», op. cit., p. 204-207 ; D. Meeks, "Génies, ... », op. cit., p. 21 ; Paul John Frandsen, «Faeces of Creator or the Temptations of the Dead », dans P. Kousoulis (éd.), op. cit., p. 25-26 ; H. te Velde, op. cit., col. 980 ; K. Szpakowska, op. cit., p. 799. 
égyptienne telle qu'elle est représentée dans le chapitre 125 du Livre des Morts, il sera question d'établir si l'aspect terrifiant d'un dieu, sa dénomination dangereuse ou sa double nature, qui consiste à protéger une personne et à punir ses ennemis dans le monde inférieur ${ }^{11}$, sont suffisants pour témoigner de sa nature diabolique.

L'étude de la scène égyptienne du jugement se fondera sur les documents les plus anciens remontant au Nouvel Empire, comme le papyrus d'Ani (fig. 1), tout en signalant ses mutations majeures. La comparaison avec les acteurs du Jugement dernier chrétien reposera sur les passages des écritures saintes qui évoquent ce thème ainsi que sur les représentations figurées de la psychostasie en Occident à partir du $\mathrm{x}^{\mathrm{e}}$ siècle $^{12}$. Quant aux influences de la représentation égyptienne de la pesée du cœur sur le Jugement dernier, déjà très étudiées ${ }^{13}$, elles dépassent le cadre de la présente étude.

11. D. Meeks, « Demons », op. cit., p. 375 ; D. Meeks, « Génies, ... », op. cit., p. 47-48 ; R. Lucarelli, «Demons in the Book of the Dead», op. cit., p. 208-211; P. Kousoulis, op. cit., p. XI-XII, XIV.

12. André Vanrie, «L'ange à la balance », dans Mina Martens, André Vanrie, Michel de Waha (éd.), Saint Michel et sa symbolique. Ouvrage publié par la Ville de Bruxelles dans le cadre de son millénaire, à l'occasion de l'exposition «Saint Michel et sa symbolique ", Bruxelles, 1979, p. 30.

13. Alfred Maury, « Recherches sur l'origine des représentations figurées de la psychostasie ou pèsement des âmes et sur les croyances qui s'y rattachaient. Deuxième article »,Revue d'Archéologie 1, 1844,p. 291-307 ; Siegfried Morenz, «Rechts und Links im Totengericht ", Zeitschrift für Ägyptische Sprache und Altertumskunde 82, 1957, p. 70, nº 6 ; Bengt Julius Peterson, « Der Totenfresser in den Darstellung der Psychostasie des altägyptischen Totenbuches », Orientalia Suecana 10, 1961, p. 39 ; Pierre Du Bourguet, «Origines lointaines d'images de saint Michel », dans Marcel Baudot (éd.), Millénaire monastique du Mont Saint Michel, Tome 3. Culte de Saint Michel et pèlerinages au Mont, Paris, 1971, p. 37-38 ; Jean Fournée, «L'Archange de la mort et du jugement », dans Marcel Baudot (éd.), op. cit., p. 67, 72, 76-77 ; A. Vanrie, op. cit., p. 18, 23 ; Erich Winter, « Der Zielpunkt bedingt die Darstellung : altägyptische und mittelalterliche Typisierung der Wäge-Szene beim Totengericht », dans Renate Brandscheidt, Theresia Mende (éd.), Schöpfungsplan und Heilsgeschichte : Festschrift für Ernst Haag zum 70. Geburtstag, Trier, 2002, p. 332-335; Jan Assmann, L'Égypte ancienne : entre mémoire et science, Paris, 2009, p. 72, $79,81$. 


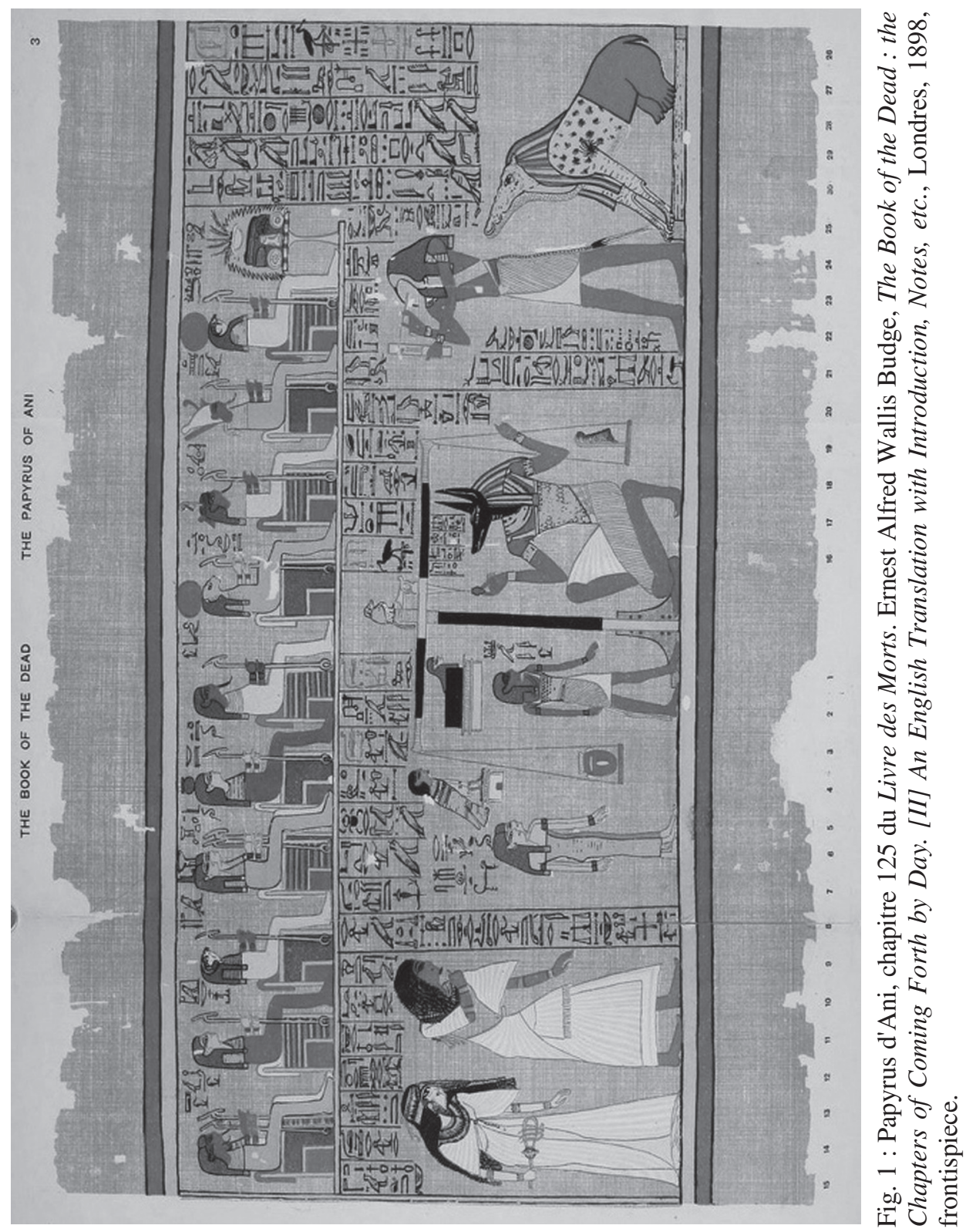




\section{LE JUGE ET LES ASSESSEURS}

\subsection{Le juge suprême : un dieu / Dieu}

La présidence du tribunal n'a pas toujours été l'apanage d'Osiris. La primauté de Rê, que reflètent les textes les plus anciens et, par la suite, la figure dominante d'Osiris dans la pesée du cœur, ont été expliqués par l'expansion du mythe de ce dernier ${ }^{14}$. Or, cette situation peut être éclairée par la présidence du dieu solaire au premier tribunal ${ }^{15}$ qui a condamné Seth pour le meurtre de son frère, ce dernier ne pouvant donc pas figurer dans l'assemblée judiciaire. À la différence du modèle mythique précédent, la version classique du jugement tend à concilier les deux juges qui voient leurs personnalités liées sous la forme de Rê-Osiris ou dans une théologie développant leur complémentarité : le processus de la pesée se déroule sous l'égide d'Osiris et le défunt justifié est ensuite introduit auprès de $\mathrm{R}^{16}$. Dans quelques cas, des concepts anciens ou locaux du jugement ont pu régir le choix d'un autre juge suprême, comme, par exemple, le roi divinisé Amenhotep I à Deir el-Médina ${ }^{17}$.

Dans la psychostasie chrétienne, la place du juge suprême est naturellement réservée au Christ, dont l'effigie surplombe la scène ${ }^{18}$ (fig. 2 : registre supérieur).

14. J. Yoyotte, op. cit., p. 41-42, 61.

15. Sur ce tribunal originel, R. Grieshammer, op. cit., p. 43-45 ; Jan Assmann, Images et rites de la mort dans l'Égypte ancienne. L'apport des liturgies funéraires, Paris, 2000, p. 63-64 ; Bernard Mathieu, «Un épisode du procès de Seth au tribunal d'Héliopolis (Spruch 477, Pyr. § 957a-959e)», Göttinger Miszellen 164, 1998, p. 71-78; Susanne Bickel, « Héliopolis et le tribunal des dieux », dans Catherine Berger, Bernard Mathieu (éd.), Études sur l'Ancien Empire et la nécropole de Saqqâra dédiées à Jean-Philippe Lauer, Orientalia Monspeliensia 9, 1997, p. 113-122.

16. C. Seeber, op. cit., p. 121-123, no 497 ; J. Yoyotte, op. cit., p. 41-42 ; Jan Zandee, Death as an Enemy, Studies in the History of Religions V, Leiden, 1960, p. 32, no 7 ; S. Bickel, op. cit., p. 114.

17. C. Seeber, op. cit., p. 131-132, n ${ }^{\circ} 580$; Bernard Bruyère, « Rapport sur les fouilles de Deir el Médineh (1927) », Fouilles de l'Institut français d'archéologie orientale V, 1928, p. 71-72, fig. 49.

18. A. Vanrie, op. cit., p. 28 ; Michel Berger, André Jacob, La Chiesa di S. Stefano a Soleto : tradizioni bizantine e cultura tardogotica, Lecce, 2007, p. $69-70$, fig. 40 . 


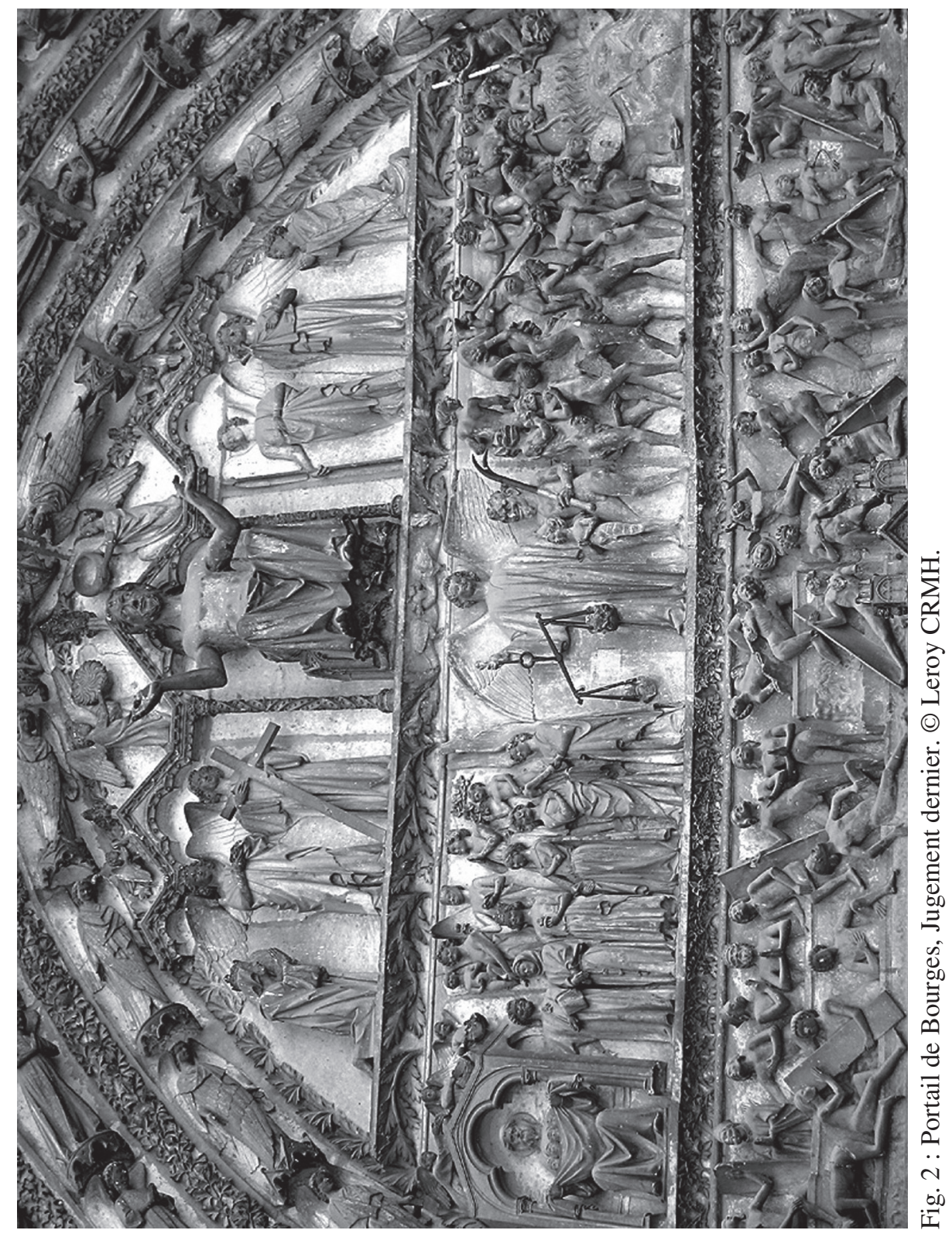




\subsection{L'entourage familial du juge suprême : des dieux à la fonction de génies protecteurs / la Vierge et Jean-Baptiste}

Osiris est souvent suivi d'Isis, sa femme, et de Nephthys, sa soeur, qui lui garantissent protection et renaissance ${ }^{19}$. Se substituant à elles, d'autres déesses, investies des mêmes fonctions, font dès lors partie de la « Sphère des Siens $»^{20}$.

Surgissant d'un lotus, les quatre enfants d'Horus peuvent se dresser juste devant le dieu des morts. Cette proximité iconographique souligne leur lien étroit avec lui. Enfantés d'une forme d'Osiris, Horus l'Ancien ${ }^{21}$, et d'Isis, ils veillent à l'intégrité physique du dieu et à sa résurrection ${ }^{22}$ par la quête et la reconstitution des morceaux dispersés du corps osirien massacré par $\operatorname{Seth}^{23}$ et par la restitution de ses organes lors de l'embaumement.

L'action d'Isis, de Nephthys ainsi que des quatre enfants d'Horus se borne à la sauvegarde d'Osiris. Le rapport de l'entourage osirien avec tout autre défunt est donc « indirect ». Ce n'est qu' au terme de la psychostasie, lorsque le mort est proclamé juste, qu'il bénéficiera, à l'instar d'Osiris, des soins prodigués par ces divinités. Aux côtés du dieu des morts, ces divinités revêtent donc le rôle de génies protecteurs.

Dans la psychostasie chrétienne, l'entourage familial de Jésus, à savoir la Vierge et Jean-Baptiste, joue, par contre, un rôle « direct » comme intercesseurs ${ }^{24}$ (fig. 2 : registre supérieur).

19. Herman te Velde, «Some Remarks on the Structure of Egyptian Divine Triads », Journal of Egyptian Archaeology 57, 1971, p. 84-86 ; Claude Traunecker, Les dieux de l'Égypte, Que sais-je? 1194, Paris, 1992, p. 62-63.

20. Jan Assmann, Liturgische Lieder an den Sonnengott, Münchner ägyptologische Studien 19, 1969, p. 341, 342, 347-352.

21. Bernard Mathieu, « Horus : polysémie et métamorphoses », Égypte nilotique et méditerranéenne 6, 2013, p. 10, nº 84 ; Bernard Mathieu, «Les Enfants d'Horus, théologie et astronomie (Enquêtes dans les Textes des Pyramides, 1)», Égypte nilotique et méditerranéenne 1,2008, p. $9, \mathrm{n}^{\circ} 4$.

22. Matthieu Heerma van Voss, «Horuskinder», dans Wolfgang Helck, Eberhard Otto, Lexikon der Ägyptologie III, Wiesbaden, 1980, col. 53.

23. Frédéric Servajean, «Le lotus émergeant et les quatre Enfants d'Horus : analyse d'une métaphore physiologique », dans Sydney Aufrère (éd.), Encyclopédie religieuse de l'univers végétal II, Orientalia Monspeliensia XI, 2001, p. 265-269, p. 264, n 22, 271-272 ; B. Mathieu, «Les Enfants d'Horus... », op. cit., p. 10-11, $\mathrm{n}^{\circ} 9$.

24. A. Vanrie, op. cit., p. 29 ; J. Fournée, op. cit., p. 83. 


\subsection{Les assesseurs}

Les assesseurs se répartissent en deux groupes, l'ennéade et les quarante-deux dieux, qui dépendent chacun d'un juge présidant le tribunal.

\subsubsection{L'ennéade : des dieux / des saints}

Les membres de l'ennéade d'Héliopolis, qui constituent la descendance de Rê, sont souvent figurés momiformes devant ou à proximité d'Osiris ${ }^{25}$. Après avoir procédé à la pesée du cœur du défunt, Thot soumet un rapport favorable à l'ennéade qui entérine cet avis, après quoi le verdict est remis à Osiris en même temps que le défunt lui est présenté ${ }^{26}$. La soumission du résultat de la pesée à ce collège divin reflète la prédominance du dieu solaire ${ }^{27}$ et de son entourage dans le précédent mythique du jugement ${ }^{28}$. L'ordre et le nombre des membres de l'ennéade varient, pouvant comprendre d'autres divinités comme Hou, Sia, Hathor ou des rois ancêtres divinisés, afin d'intégrer le panthéon du pays tout entier dans la sentence et de lui conférer un caractère universel ${ }^{29}$.

Excepté le caractère agressif de quelques membres de l'ennéade ${ }^{30}$ (infra 1.3.2), celle-ci peut être mise en parallèle avec les assesseurs correspondant aux saints ${ }^{31}$ qui encadrent le Christ et assistent à la psychostasie (Matthieu 19, 28 ; 1 Corinthiens 6, 2-3).

1.3.2. Les quarante-deux assesseurs : des démons ou des génies exécuteurs ? / des anges exécuteurs

Le deuxième groupe d'assesseurs est constitué de quarante-deux divinités que préside « le grand dieu, le seigneur des deux Maât » ${ }^{32}$, siégeant dans la Salle de ces deux déesses qui incarnent la norme

25. C. Seeber, op. cit., p. 135.

26. Ibid., p. 135 ; J. Yoyotte, op. cit., p. 47-48.

27. C. Seeber, op. cit., p. 133 ; J. Yoyotte, op. cit., p. 49.

28. Sur le précédent mythique, supra $\mathrm{n} .15$.

29. S. Bickel, op. cit., p. 115, $\mathrm{n}^{\circ} 14$; Erik Hornung, Conceptions of God in Ancienl Egypt, Londres, 1982, p. 222-223 ; C. Seeber, op. cit., p. 135.

30. J. Spiegel, op. cit., p. 47 ; chapitre 17 du Livre des Morts, C. Seeber, op. cit., p. 130-131, n. 569-571.

31. A. Vanrie, op. cit., p. 29.

32. J. Yoyotte, op. cit., p. 51-52 (trad.), 59; C. Maystre, Les déclarations d'innocence (Livre des Morts, chapitre 125), Recherches d'Archéologie, de Philologie et d'Histoire 8, 1937, p. 13 (texte hiéroglyphique), 17 (traduction). 
d'équité. Cette partie du jugement, connue sous le nom de la « confession négative », se déroule « devant Ounenenefer ${ }^{33}$, ce qui indique l'identité osirienne du président. Pendant la séance, le mort est censé connaître les noms des quarante-deux divinités et nier des péchés concernant les paroles et les actes ${ }^{34}$. La comparution du défunt devant cette assemblée vise à déployer son innocence dans les quarante-deux nomes du territoire égyptien, représentés chacun par un dieu ${ }^{35}$.

La situation des deux collèges divins et les textes qui accompagnent leurs effigies peuvent expliquer la différence de leurs interventions dans le jugement et les catégories du divin auxquelles ils appartiennent. Sujette à controverse, la fonction judiciaire des quarante-deux divinités a été perçue comme contournable magiquement par les énoncés du défunt ${ }^{36}$, idée réfutée par d'autres chercheurs qui plaident en faveur d'une application de la justice en vigueur ${ }^{37}$. Jan Assmann reconnaît dans l'introduction du mort devant les quarante-deux assesseurs et dans la pesée du cour devant l'ennéade deux examens distincts, le premier «extérieur» de ce que dit la bouche, le second «intérieur» du cœur de l'homme ${ }^{38}$. Cette interprétation, qui met en valeur le principe de l'équité, peut être appuyée par la situation du collège des quarante-deux dans la scène ainsi que par les légendes accolées à leurs effigies. Situés à proximité des portes de la Salle des deux Maât au Nouvel Empire, les quarante-deux assesseurs autorisent le défunt à y pénétrer s'il connaît leurs noms et s'il nie avoir commis des péchés. Ces conditions d'entrée, en particulier la récitation de leurs dénominations et l'interrogatoire final du chapitre 125 que subit le

33. J. Yoyotte, op. cit., p. 51-52 (trad.), 59 ; C. Maystre, op. cit., p. 16 (texte hiéroglyphique), 17 (traduction).; C. Seeber, op. cit., p. 137.

34. Sur la traduction et l'analyse de l'ensemble des péchés: J. Yoyotte, op. cit., p. 52-54, 59-60 ; J. Assmann, Mort et au-delà, op. cit., p. 132-133; J. Assmann, Maât, op. cit., p. 76-79 ; Charles Maystre, op. cit., p. 23-55, 65-103; James Henry Breasted, Development of Religion and Thought in Ancient Egypt, New York, 1959, p. 299-304; M. Lichtheim, op. cit., p. 103-144.

35. J. Assmann, Mort et au-delà, op. cit., p. 136 ; J. H. Breasted, op. cit., p. 302.

36. J. Yoyotte, op. cit., p. 19 ; J. H. Breasted, op. cit., p. 307.

37. J. Assmann, Mort et au-delà, op. cit., p. 133-134.

38. Jan Assmann, «Zur Geschichte des Herzens im Alten Ägypten », dans Jan Assmann, Theo Sundermeier (éd.), Die Erfindung des inneren Menschen. Studien zur religiösen Anthropologie, Gütersloh, 1993, p. 89-90. 
mort ${ }^{39}$, font de ces dieux des gardiens de portes analogues à ceux qui sauvegardent les accès au monde inférieur. L'intervention de ce collège divin dans le jugement précède la pesée de son cœur et ne lui est donc pas simultanée, au contraire de ce que l'on a suggéré auparavant $t^{40}$.

Toutefois, la représentation des déclarations d'innocence et de la pesée du cœur subit une modification majeure à l'époque tardive : les deux événements apparaissent dans une seule et même scène, dans laquelle les quarante-deux assesseurs prennent la place de l'ennéade ${ }^{41}$. Ce changement figuratif témoigne-t-il de la simultanéité des deux actions ? Deux arguments plaident en faveur d'une réponse négative. Le premier réside dans les conventions de l'art égyptien, selon lesquelles deux moments différents de la vie peuvent être figurés dans une seule scène. Par exemple, dans une même procession funéraire, une épouse peut être dessinée vivante, près du corps de son époux, et momiforme derrière la dépouille de son mari ${ }^{42}$. Le second argument se trouve dans la scène même : une petite figure du défunt se présente devant les quarante-deux divinités, alors qu'une grande effigie du même personnage se trouve debout devant la balance ${ }^{43}$, ce qui prouve l'indépendance et la succession des deux actions, comme dans les représentations les plus anciennes de la psychostasie. L'introduction du mort devant les quarante-deux assesseurs n'est guère un moyen "magique ». Elle constitue plutôt une phase liminaire pour franchir le premier seuil du jugement. Cette étape s'avère « théorique » et cruciale car il serait inconcevable d'évaluer, au moyen de la balance, la conformité d'un homme avec Maât, si celui-ci ignore ses règles. Cette phase de connaissance «théorique » des lois de la justice, que contrôlent les quarante-deux dieux, est suivie d'une phase «pragmatique »,

39. J. Yoyotte, op. cit., p. 56 (trad.); Edouard Naville, Das aegyptische Todtenbuch der XVIII. bis XX. Dynastie aus verschiedenen Urkunden, Band I, Berlin, 1886, pl. 137-138 (texte hiéroglyphique).

40. J. Assmann, Mort et au-delà, op. cit., p. 167.

41. C. Seeber, op. cit., p. 138, fig. 23-26.

42. Petra Barthelmess, Der Übergang ins Jenseits in den thebanischen Beamtengräbern der Ramessidenzeit, Studien zur Archäologie und Geschichte Altägyptens 2, 1992, p. 99 ; Heinrich Schäfer, Emma Brunner-Traut (éd.), Principles of Egyptian Art, translated and edited, with an introduction, by John Baines, Oxford, 1974, p. 160-162, 227-230.

43. C. Seeber, op. cit., fig. 23-26. 
durant laquelle l'ennéade vérifie la mise en pratique des principes de Maât.

Les figures hybrides des quarante-deux divinités armées de couteaux ainsi que leurs noms redoutables, comme « celui qui avale les ombres $»^{44}$, leur ont valu la qualification de démons ${ }^{45}$. Ce même caractère terrifiant se rencontre chez certains dieux pouvant s'ajouter à l'ennéade ou au collège des quarante-deux, comme les quatre enfants d'Horus ${ }^{46}$. Un passage du chapitre XVII du Livre des Morts précise que ceux-ci, qui constituent le tribunal d'Osiris, font « carnage aux malfaiteurs $»^{47}$. Le caractère dangereux des quarante-deux assesseurs ne permet pas de déterminer leur nature démoniaque. En effet, ils n'accomplissent aucun acte contraire à la justice et agissent en conformité avec Maât, dont l'emblème, la plume, peut surmonter leur tête ${ }^{48}$. Ils sont plutôt des gardiens de portes, des «bourreaux $»^{49}$ ou des génies qui exécutent les coupables comme le fait la dévoreuse (infra 2.3).

La fonction des quarante-deux dieux, de même que celle de la dévoreuse, rappelle le rôle des anges qui châtient le diable et ses troupes dans la psychostasie chrétienne (infra 2.3).

\section{LeS AUXILIAIRES DE LA PESÉE}

Quand il se dirige vers la balance, le défunt peut être accompagné de son $b a$ (fig. 1) et de son ombre illustrée par une silhouette noire. Ces composantes du corps de la personne semblent prendre part à sa récompense ou à sa condamnation. Le $b a$ du mort justifié survit en se déplaçant d'un monde vers un autre ${ }^{50}$. Dans le cas contraire,

44. J. Yoyotte, op. cit., p. 53 (trad.) ; C. Maystre, op. cit., p. 68 (texte hiéroglyphique).

45. C. Seeber, op. cit., p. 135-137 ; J. Spiegel, op. cit., p. 47.

46. C. Seeber, op. cit., p. 130-131, $\mathrm{n}^{\circ}$ 569-570; J. Spiegel, op. cit., p. 47.

47. C. Seeber, op. cit., p. 130-131, n ${ }^{\circ} 571$; Hermann Grapow, Religiöse Urkunden. Ausgewählte Texte des Totenbuches (Heft 1-3), Urkunden des ägyptischen Altertums V, 1915-1917, p. 42, 1. 4 (texte hiéroglyphique); Erik Hornung, Das Totenbuch der Ägypter, Zürich, München, 1979, p. 66, 1. $149-153$ (trad.).

48. C. Seeber, op. cit., p. 130-131, no 136, no 601.

49. Terme emprunté à J. Yoyotte, op. cit., p. 59-60.

50. J. Assmann, Maât, op. cit., p. 72-73. 
il est voué à la seconde mort, de même que les autres éléments de la personnalité ${ }^{51}$. Leur récompense ou leur condamnation semble indiquer que ceux-ci attendent un sort associé au résultat du jugement.

La scène traditionnelle de la psychostasie évoque la pesée du cœur du mort devant la plume de Maât qui sert d'étalon, mais il existe plusieurs variantes dans lesquelles d'autres figures occupent les deux plateaux de la balance ${ }^{52}$. Si, pendant sa vie, le défunt a suivi les consignes de son cœur juste, ce dernier n'est pas censé témoigner contre lui pendant le jugement ${ }^{53}$. La justification de la personne réside alors dans l'équilibre des deux plateaux de la balance, témoignant de la soumission du défunt aux principes de Maât pendant son existence terrestre. Par ailleurs, l'image conventionnelle de la psychostasie, opposant le cœur à la plume de Maât, ne précise rien de «concret » quant à la pesée des actions ${ }^{54}$ que mentionne l'enseignement de Mérikarê : « quand l'homme sera de reste après la mort (litt. "le débarquement"), ses actions seront déposées à côté de lui comme une somme (spp s m-hht mny.t rdi.w sp.f r-gs.f $m$ ' $h \cdot w) \gg^{55}$. La divergence entre cette dernière source littéraire et l'iconographie du jugement a été interprétée comme témoignant d'un affinement du sentiment éthique ${ }^{56}$. Toutefois,

51. J. Zandee, op. cit., p. 14, 188, no 8 ; Joseph Vergote, «Immortalité conditionnée de l'âme ou survie inconditionelle dans l'Égypte ancienne », dans Aristide Théodoridès, Paul Naster, Julien Ries, Vie et survie dans les civilisations orientales, Acta Orientalia Belgica 3, Louvain, 1983, p. 65-69, p. 66-68.

52. Sur les différentes versions de la psychostasie: C. Seeber, op. cit., p. 74-83 ; Philippe Derchain, «L'CEil, Gardien de la Justice», Zeitschrift für Ägyptische Sprache und Altertumskunde 83, 1958, p. 75-76; H. Gaber, « Deux variantes...», op. cit., p. 1-16.

53. J. Assmann, «Zur Geschichte des Herzens...», op. cit., p. 100 ; J. Assmann, Mort et au-delà, op. cit., p. 167 ; C. Seeber, op. cit., p. 76.

54. C. Seeber, op. cit., p. 77, 81.

55. Traduction de J. Assmann, Images et rites, op. cit., p. 62 ; Wladimir Golénischeff, Les papyrus hiératiques $N^{\mathrm{o}} 1115,1116$ A et $1116 \mathrm{~B}$ de l'Ermitage impérial à St-Pétersbourg, St. Pétersbourg, 1913, pl. 10, X (1. 55) (textes hiéroglyphique et hiératique); Joachim Friedrich Quack, Studien zur Lehre für Merikare, Göttinger Orientforschungen 4, 23, 1992, p. 175 (55) (M) (texte hiéroglyphique), p. 34-35 ; Dieter Müller, « Grabausstattung und Totengericht in der Lehre für König Merikare », Zeitschrift für Ägyptische Sprache und Altertumskunde 94, 1967, p. 117 (1. 54-56 : texte hiéroglyphique), p. 118, 123 (traduction).

56. Hans Bonnet, Reallexikon der ägyptischen Religionsgeschichte, Berlin, 1952, p. 341 ; Erich Lüddeckens, "Alter und Einheitlichkeit der ägyptischen Vorstellung vom Totengericht», dans Akademie der Wissenschaften und der Literatur, Wiesbaden, 1953, p. 199, n'est pas d'accord avec cette idée. 
l'évaluation du cœur, agissant comme la conscience ${ }^{57}$, correspond à l'examen des actions ${ }^{58}$. La pesée du coeur présente donc une version figurative et métaphorique de celle des bons et des mauvais actes.

\subsection{Les peseurs : les dieux juges Thot, Horus, Anubis et Maât / des anges tenant la balance ou préparant le Jugement dernier}

Thot, Horus, Anubis et Maât peuvent conduire le mort devant la balance, contrôler sa pesée ou l'introduire devant Osiris. Le choix de l'un ou l'autre d'entre eux varie selon les périodes et les lieux ${ }^{59}$. Par exemple, Maât tient le peson pour vérifier la stabilité de la balance à Deir el-Médina au Nouvel Empire, alors qu'ailleurs, elle assiste passivement au déroulement du jugement ${ }^{60}$. Malgré leur interaction, Thot, Horus et Anubis se chargent de tâches spécifiques pendant la psychostasie. L'intervention de Thot, qui a jugé Horus et Seth dans le tribunal mythique, le prédispose à exercer l'autorité de juge ${ }^{61}$, de même que son association étroite aux mathématiques et à l'écriture l'amène à procéder à l'enregistrement écrit du jugement et à remettre celui-ci à l'ennéade ${ }^{62}$. Lorsque le défunt est introduit par Horus auprès d'Osiris, il aspire à la même justification dont s'est targué Horus contre Seth ${ }^{63}$. Quant à Anubis, il rend au mort son cœur une fois le verdict positif promulgué, acte qui s'explique par ses soins d'embaumeur ${ }^{64}$.

L'idée selon laquelle la fonction du juge et sa connaissance du passé et des fautes du défunt suscitent la crainte de celui-ci ${ }^{65}$ transparaît principalement dans deux sources. La première, un extrait des Textes des Sarcophages, décrit une confrontation entre

57. Michel Malaise, Les scarabées de cour dans l'Égypte ancienne, Monographies Reine Elisabeth 4, Bruxelles, 1978, p. 11 ; Alexandre Piankoff, Le « cœur » dans les textes égyptiens depuis l'Ancien jusqu'à la fin du Nouvel Empire, Paris, 1930, p. 82 ; J. Assmann, «Zur Geschichte des Herzens... », op. cit., p. 99 ; J. Yoyotte, op. cit., p. 41.

58. M. Lichtheim, op. cit., p. 125.

59. C. Seeber, op. cit., p. 139-162 ; J. Yoyotte, op. cit., p. 45 ; J. G. Griffiths, op. cit., p. 227 ; J. Assmann, Mort et au-delà, op. cit., p. 130.

60. C. Seeber, op. cit., p. 140, fig. 51.

61. S. Bickel, op. cit., p. 115 ; J. Assmann, Images et rites, op. cit., p. 63-64.

62. C. Seeber, op. cit., p. 147-148, 151 ; J. Yoyotte, op. cit., p. 45.

63. C. Seeber, op. cit., p. 159.

64. Ibid., p. 154-156.

65. J. Assmann, Images et rites, op. cit., p. 65. 
le mort et le juge : «Salut à toi Thot... Tu sièges contre moi à leur tête (hms.k r.i m-hnt.sn) (c.-à-d. à la tête du grand tribunal) ${ }^{66}$. La seconde, extraite de l'enseignement de Mérikarê, met en garde la destinataire contre la sévérité du juge à cause de son savoir : «Funeste est l'accusateur, étant quelqu'un qui sait ( $q s n$ pw srh.y $m$ s33) ${ }^{67}$. Se basant sur ce dernier passage, J. Assmann estime que « le mort n'est confronté ni à Seth ni à des ennemis potentiels, mais à un accusateur divin et omniscient, devant lequel il doit prouver son innocence ${ }^{68}$, ce qui induirait en confusion tout à la fois ses adversaires éventuels, Seth et le juge. Or, seul ce dernier représente l'instance judiciaire que le défunt doit convaincre.

Une divinité qui surveille la pesée conduit à penser au rôle de l'Archange Michel comme, par exemple, dans le portail de la cathédrale de Bourges (XIII ${ }^{\mathrm{e}}$ siècle) ${ }^{69}$ (fig. 2 : registre médian) ou le panneau central du triptyque du Jugement dernier de Hans Memling ( $\mathrm{XV}^{\mathrm{e}}$ siècle), conservé au Musée de Gdansk ${ }^{70}$. Cette image bien connue de «l'ange à la balance » est ancrée dans des traditions antiques puisant leur origine dans la psychostasie égyptienne ${ }^{71}$. Le rôle des anges chargeant les plateaux de la balance est immortalisé tant dans l'iconographie que dans l'hagiographie occidentale ${ }^{72}$, alors qu'en Orient il ne se rencontre que dans les sources écrites, comme par exemple dans la vie de Saint Pierre l'Ascète, honoré dans

66. Adriaan de Buck, The Egyptian coffin texts VI, Oriental Institute Publications 81, 1956, p. 209, g, formule 587; traduction de S. Bickel, op. cit., p. 115, no 19 ; Raymond Oliver Faulkner, The Ancient Egyptian Coffin Texts. Volume II, Warminster, 1973, p. 190 (trad.).

67. Traduction de J. Assmann, Images et rites, op. cit., p. 62 ; W. Golénischeff, op. cit., pl. 10, X (1. 54) (textes hiéroglyphique et hiératique) ; J. F. Quack, op. cit., p. 174 (54) (E) (texte hiéroglyphique), p. 34-35 (translittération et traduction).

68. J. Assmann, Images et rites, op. cit., p. 62.

69. Béatrice de Chancel-Bardelot, Dictionnaire de la cathédrale de Bourges, suivi d'un répertoire des saints dans la cathédrale, Dijon, 2008, p. 158-159; Laurence Brugger, Yves Christe, Bourges : la cathédrale, [Saint-Léger-Vauban], 2000, p. 284-312, fig. 110, 113.

70. Jacques Le Goff, Un Moyen Âge en images, Paris, 2007, p. 173, fig. 101.

71. A. Maury, op. cit., p. 300-307 ; Alfred Maury, « Des divinités et des génies psychopompes dans l'antiquité et au moyen âge », Revue d'Archéologie 2, 1845, p. 501, 503, 506 ; J. Fournée, op. cit., p. 72, 76-78 ; P. Du Bourguet, op. cit., p. 37-38 ; A. Vanrie, op. cit., p. 30-31 ; E. Winter, op. cit., p. 332-335.

72. <http://coptica.free.fr/michel_1_archange_chevalier_1040.htm> ; Alfred Maury, «Recherches sur l'origine des représentations figurées de la psychostasie ou pèsement des âmes et sur les croyances qui s'y rattachaient. Premier article », Revue d'Archéologie 1, 1844, p. 235-249; voir supra n. 13, 70. 
l'église égyptienne ${ }^{73}$. D'une façon générale, la fonction de Thot, qui soumet par écrit le résultat de la pesée, est plus déterminante que celle des anges dans les écritures saintes. Ceux-ci protègent le trépassé $^{74}$ et assistent à Dieu pendant le Jugement dernier (Matthieu $24,31 ; 25,31)^{75}$.

\subsection{Meskhenet, Chaï, Renoutet : des dieux qui agissent comme témoins ou qui récompensent ?}

Meskhenet, Chaï et Renoutet personnifient la naissance, le destin et la durée de vie de l'homme ainsi que sa croissance vitale ${ }^{76}$ (fig. 1). Étroitement liées à la venue au monde de tout individu, les trois divinités peuvent être évoquées dans la psychostasie sous la forme de briques de naissance à tête humaine ${ }^{77}$. L'identification de leur rôle dans le jugement soulève encore des questions car très peu de textes l'abordent. Cet ensemble divin peut intégrer la personnalité de l'individu dans une procession d'offrandes accordées «... pour son Chaï, pour son temps de vie, pour sa Meskhenet, pour

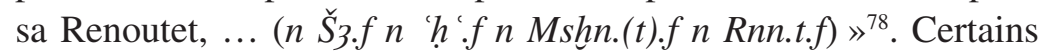
voient en ces composantes de la personne des témoins à décharge qui accréditent le défunt face à la norme de Maât, en faisant prendre en compte les joies, les peines et d'autres circonstances de

73. Synaxaire copte du 25 Touba : De Lacy O'Leary, The Saints of Egypt : an Alphabetical Compendium of Martyrs, Patriarchs and Sainted Ascetes in the Coptic Calendar, Commemorated in the Jacobite Synaxarium, Amsterdam, 1974, p. 227 ; traduction française d'une version arabe plus détaillée de cette vie : http://www.eglise-copte-orthodoxe-de-france.fr/pdf/synaxaire/25_05. pdf

74. A. Maury, «Des divinités et des génies... », op. cit., p. 507-520; J. Fournée, op. cit., p. 66-67, 70-72 ; A. Vanrie, op. cit., p. 22.

75. J. Fournée, op. cit., p. 74 ; Henri Cazelles, «Le jugement des morts en Israël », dans Le jugement des morts, Sources Orientales 4, Paris, 1961, p. 135-136 ; A. Vanrie, op. cit., p. 28-29: transposition en images de la préparation du trône divin (Apocalypse 20, 11-12) et de la participation des anges.

76. C. Seeber, op. cit., p. 85-86 ; J. G. Griffiths, op. cit., p. 226-227.

77. C. Seeber, op. cit., p. 85-86, 134, fig. 49 ; Jan Quaegebeur, Le dieu égyptien Shaï dans la religion et l'onomastique, Orientalia Lovaniensia Analecta 2, 1975, p. 153.

78. Wolfgang Helck, Urkunden der 18. Dynastie IV, Leipzig, 1909, p. 1060, 1. 12-13 (texte hiéroglyphique); J. Quaegebeur, op. cit., p. 133, nº 1 (trad.), 119, 146 ; C. Seeber, op. cit., p. 86, no 320 ; Jan Assmann, « Die Inschrift auf dem äußeren Sarkophagdeckel des Merenptah», Mitteilungen des Deutschen Archâologischen Instituts Abteilung Kairo 28, 1, 1972, p. 50-51, 1. 35-37, p. 61 (19). 
son existence terrestre ${ }^{79}$. Dans la psychostasie, les noms des trois divinités sont cependant reproduits sans le pronom possessif qui les associe au défunt ${ }^{80}$, ce qui laisse supposer qu'elles exercent sans doute leur fonction divine en accomplissant la renaissance ainsi que le renouvellement du maintien en vie et de la durée de vie. L'association de briques de naissance à ces dieux, exprimant le désir du mort de renaître ${ }^{81}$, semble renforcer cette hypothèse. Les trois divinités marqueraient alors la récompense en opposition au châtiment que concrétise la dévoreuse, nommée parfois Chayt ${ }^{82}$, ce qui est précisément l'équivalent féminin de Chaï.

Incarnant la renaissance et le maintien de la vie du défunt, ces divinités de nature particulière ne peuvent être rapprochées d'aucun intervenant dans le jugement chrétien.

\subsection{Les exécuteurs du châtiment : la dévoreuse, un démon ou un génie exécuteur ? / des anges exécuteurs}

Près de la balance se trouve la dévoreuse Âmmout, dont le nom est dérivé du verbe «avaler ( $m$ ) » ${ }^{83}$. Elle est censée engloutir le cœur du mort condamné par le tribunal. Son aspect monstrueux, hybride du crocodile, de l'hippopotame et du lion, a incité à reconnaître en elle une figure démoniaque, à l'instar d'Apophis ${ }^{84}$. Bien au contraire de ce dernier, l'ennemi par excellence du soleil, la dévoreuse, de même que les quarante-deux assesseurs (supra

79. C. Seeber, op. cit., p. 86 ; J. Yoyotte, op. cit., p. 46 ; J. Quaegebeur, op. cit., p. 147-149; John Baines, Fecundity Figures : Egyptian Personification and the Iconology of a Genre, Warminster, 1985, p. 63-64 ; J. Assmann, « Die Inschrift », op. cit., p. 61 (19), n 40 ; Marc Gabolde, «Une interprétation alternative de la "pesée du cœur" du Livre des Morts », Égypte, Afrique \& Orient 43, octobre 2006, p. 18-19.

80. Voir, par exemple, C. Seeber, op. cit., p. 134, fig. 49, p. 85, fig. 26.

81. C. Seeber, op. cit., p. 88 ; J. Assmann, « Die Inschrift », op. cit., p. 50-51, 1. 35-37, p. 61 (19) ; J. G. Griffiths, op. cit., p. 226-227, no 97-98; J. Baines, op. cit., p. 64.

82. C. Seeber, op. cit., p. 170-171.

83. Sur les différentes écritures de son nom, voir C. Seeber, op. cit., p. 167-171.

84. Ibid., p. 164, 172-173 ; Henry George Fischer, « The Ancient Egyptian Attitude Towards the Monstrous », dans Ann Elizabeth Farkas, Prudence Oliver Harper, Evelyn Byrd Harrison (éd.), Monsters and Demons in the Ancient and Medieval Worlds. Papers Presented in Honor of Edith Porada, Mainz, 1987, p. 20 ; R. Lucarelli, «Demons (Benevolent...)», op. cit., p. 1-10: <http:// escholarship.org/uc/item/1r72q9vv>; R. Lucarelli, «Demons in the Book of the Dead», op. cit., p. 207 ; H. te Velde, op. cit., col. 980. 
1.3.2), n'évoque pas le mal en soi. Elle assume plutôt les directives de Maât ${ }^{85}$, telle un bourreau ${ }^{86}$. Son appartenance à la justice est pleinement confirmée par la plume de Maât dont elle est coiffée à partir de la $22^{\mathrm{e}}$ dynastie ${ }^{87}$. La figure du monstre en action se livrant à l'anéantissement des ombres des malfaiteurs, et non pas à celle du défunt, n'est attestée qu'à l'époque grécoromaine ${ }^{88}$. Représenté sur un socle auprès d'Osiris à l'époque saïte, l'animal hybride, outre sa dimension punitive, acquiert le statut de gardienne des portes du monde inférieur ${ }^{89}$. Ce rôle positif de la déesse remonte sans doute au Nouvel Empire puisque Toutânkhamon est qualifié, sur un de ses lits funéraires, de «l'aimé d'Âmmout ('mr(y) '\{3\}mm.t)» ${ }^{90}$.

Dans le pendant chrétien de la psychostasie, à quelle catégorie d'êtres pourrait-on comparer la dévoreuse, ainsi que les quarante-deux assesseurs (supra 1.3.2) ? Si, au premier abord, le démon paraît être une réponse évidente, la question s'avère plus difficile à cerner au regard des sources iconographiques et textuelles. L'iconographie du Moyen Âge occidental a réservé une place prépondérante au diable et à ses alliés comme tortionnaires et exécuteurs des malfaiteurs dans le but d'inciter à la crainte ${ }^{91}$ (fig. 2 : registre médian, côté gauche). Or, les écritures soulignent le sort passif du diable, châtié par les anges, comme dans la parabole du bon grain et de l'ivraie: « De même que l'on ramasse l'ivraie pour la brûler au feu, ainsi en sera-t-il à la fin du monde : le Fils de l'homme enverra ses anges ; ils ramasseront, pour les mettre hors de son Royaume, toutes les causes de chute et tous ceux qui commettent l'iniquité, et ils les jetteront dans la fournaise de feu » (Matthieu, 13, 40-42) $)^{92}$. Ce

85. C. Seeber, op. cit., p. 167-175 ; J. Assmann, Mort et au-delà, op. cit., p. $128-129$.

86. E. Winter, op. cit., p. 329-330.

87. C. Seeber, op. cit., p. 165, fig. 67, pl. 26, 28.

88. Voir, à titre d'exemple, E. Winter, op. cit., p. 334, fig. 3 ; C. Seeber, op. cit., p. 171-175, 172, fig. 70, 186, fig. 79 ; Dieter Kurth, Der Sarg der Teüris. Eine Studie zum Totenglauben im römerzeitlichen Ägypten, Aegyptiaca Traverensia 6, 1990, p. 66-67, fig. 24-25.

89. B. J. Peterson, op. cit., p. 35-39 ; C. Seeber, op. cit., p. 175-184, 188, fig. 77.

90. M. Gabolde, op. cit., p. 17 (texts hiéroglyphique et traduction).

91. J. Le Goff, op. cit., p. 172-175 ; A. Vanrie, op. cit., p. 26, 28-29.

92. Camille Focant, Daniel Marguerat, Le Nouveau Testament commenté : Texte intégral. Traduction æecuménique de la Bible, Montrouge, Genève, 2012, p. 78-81 ; J. Fournée, op. cit., p. 87-88 ; A. Vanrie, op. cit., p. 22. 
passage atteste du rôle des anges comme serviteurs de la justice divine, qui assument le châtiment du diable et de ses alliés en les enflammant. La mise en parallèle des psychostasies égyptienne et chrétienne révèle que les fonctions des anges, de la dévoreuse et des quarante-deux assesseurs s'équivalent : défendant la justice, ils œuvrent tous pour le châtiment des ennemis.

\section{Les ENNEMIS : Seth ET SES ALliÉS / LE DÉMON}

Lorsque l'innocence du défunt est proclamée, celui-ci est justifié contre ses ennemis, idée majeure qui s'exprime dans le discours des divinités au terme de la psychostasie ${ }^{93}$. L'identité et la classification des ennemis du défunt et des dieux ont fait l'objet de quelques études $^{94}$. Selon le modèle mythique sur lequel se calque la psychostasie classique, Seth est « l'ennemi existentiel »d'Osiris ${ }^{95}$. En outre, d'autres adversaires peuvent intenter un procès contre le défunt au moment de l'examen de son cœur ${ }^{96}$. Ces opposants du mort sont associés aux acolytes de Seth, comme, par exemple, dans le chapitre 18 du Livre des Morts qui est centré sur la parution du défunt en justice : «Ô Thot qui as fait triompher Osiris de ses ennemis ( $h f t y . w)$, fais triompher $\mathrm{NN}$ (= le défunt) de ses ennemis (hfty.w) comme tu as fait triompher Osiris de ses ennemis (hfty.w) en présence du tribunal dans lequel est Rê et dans lequel est Osiris à Héliopolis, en cette nuit du souper, en cette nuit du combat et de la mise en garde des rebelles (sby.w), en ce jour où les ennemis ( $h f t y . w)$

93. Voir, à titre d'exemples, C. Seeber, op. cit., p. 143, nº 636 ; J. Assmann, Mort et au-delà, op. cit., p. 417 ; S. Bickel, op. cit., p. 116.

94. J. Zandee, op. cit., p. 217-224 ; J. Assmann, Images et rites, op. cit., p. $60-62$.

95. J. Assmann, Mort et au-delà, op. cit., p. 121-122; Georg Meurer, «Die Verfemung des Seth und seines Gefolges in den Pyramidentexten und in späterer Zeit », dans Heinz Felber (éd.), Feinde und Aufrührer: Konzepte von Gegnerschaft in ägyptischen Texten besonders des Mittleren Reiches, Abhandlungen der Sächsischen Akademie der Wissenschaften zu Leipzig, philologisch-historische Klasse 78, Heft 5, Leipzig, 2005, p. 175, nº 8 ; J. Zandee, op. cit., p. 219, 221-222 ; Herman te Velde, Seth, God of Confusion. A Study of his Role in Egyptian Mythology and Religion, Leiden, 1967, p. 81-82 ; Bernard Mathieu, « Seth polymorphe : le rival, le vaincu, l'auxiliaire », Égypte nilotique et méditerranéenne 4, 2011, p. 150 ; R. Grieshammer, op. cit., p. 44.

96. J. Zandee, op. cit., p. 32, 219-220 ; J. Assmann, Images et rites, op. cit., p. $60-62$. 
du maître de l'univers furent anéantis. (Gloses) En ce qui concerne le grand tribunal qui est à Héliopolis, c'est Atoum, c'est Chou, c'est Tefnout. En ce qui concerne la mise en garde des rebelles (sby.w) - c'est la destruction de la bande de Seth lorsqu'il a (voulu) répéter son crime $»^{97}$. Les deux termes « adversaires / ennemis (hfty.w)» et « rebelles $(s b y . w) »$ se présentent comme synonymes des alliés de Seth $^{98}$.

Peut-on considérer Seth, le meurtrier d'Osiris, et les autres ennemis du défunt comme des démons ? Selon J. Assmann, Seth n'incarne pas le mal absolu car il vient à être inséré dans le mythe de Rê en tant que son défenseur contre le serpent Apophis ${ }^{99}$. En effet, les actions de Seth, qui s'inscrivent dans le cadre de deux mythes fondateurs, issus de régions différentes, ont entraîné une confusion quant à l'interprétation de ses rôles : d'une part, Seth l'héliopolitain assassine son frère Osiris, d'autre part, Seth de Noubet subit une défaite lors de son conflit avec Horus de Nékhen ${ }^{100}$. Vaincu par Horus, Seth devient alors son assistant, notamment dans le combat solaire contre Apophis ${ }^{101}$. Le caractère «démoniaque » de Seth est particulièrement mis en exergue dans le Livre de la Nuit et dans celui de l'Amdouat par l'alternance de son nom avec celui d'Apophis, l'ennemi de Rê $\hat{e}^{102}$. L'opposition de ces dieux à Osiris et Rê relève d'un contraste plus large entre un dieu (ntrr) et son adversaire ( $h f t y)$ reconnu comme démoniaque pour ses actes mauvais ${ }^{103}$.

Bien que le châtiment des ennemis et des pécheurs soit bien illustré dans les livres du monde inférieur, datant du Nouvel Empire ${ }^{104}$,

97. Traduction du S. Bickel, op. cit., p. 117, nº 30 ; E. Naville, op. cit., pl. 31, col. 1-6 (texte hiéroglyphique).

98. G. Meurer, op. cit., p. 180, 184.

99. J. Assmann, Mort et au-delà, op. cit., p. 123.

100. B. Mathieu, «Seth polymorphe », op. cit., p. 137-158, particulièrement p. 149, 154 ; H. te Velde, Seth, op. cit., p. 81-84, 99-100, 71, n 2-3.

101. B. Mathieu, «Seth polymorphe», op. cit., p. 149 ; H. te Velde, Seth, op. cit., p. 71, $\mathrm{n}^{\circ} 2-3$.

102. Erik Hornung, Das Amduat. Die Schrift des verborgenen Raumes. Übersetzung und Kommentar, Ägyptologische Abhandlungen 7, 1963, Teil 1. Text, p. 8, Teil II, p. 15-16, n 37 ; Gilles Roulin, Le Livre de la Nuit. Une composition égyptienne de l'au-delà, Orbis biblicus et orientalis 147, 1-2, 1996, Partie 2, p. 76, Partie 1, p. 206-208 ; Dietrich Wildung, « Feindsymbolik », dans Wolfgang Helck, Eberhard Otto, Lexikon der Ägyptologie II, Wiesbaden, 1977, col. 147 ; G. Meurer, op. cit., p. 173.

103. H. te Velde, Seth, op. cit. p. 91-94 ; J. Zandee, op. cit., p. 224, no 4-5.

104. Par exemple, J. Zandee, op. cit., p. 18-19, 36-38. 
Seth s'est évadé dans le désert suite à la sentence énoncée contre lui ; de même, Apophis est battu, brulé et coupé en pièces, mais à chaque nuit il réapparaît à nouveau ${ }^{105}$. Leur défaite n'est donc pas définitive, mais elle est, à l'image du monde égyptien, cyclique, se renouvelant à chaque passage nocturne de Rê dans le monde inférieur, comme à chaque justification d'une personne identifiée à Osiris.

Le principe selon lequel le moteur du mal, qu'il s'agisse de Seth ou d'Apophis, demeure au monde, se rencontre dans la parabole du bon grain et de l'ivraie, qui développe la cohabitation du bien et du mal dans le monde jusqu'au Jugement dernier, moment de l'épuisement définitif de la puissance de nuisance du démon (Matthieu, 13, 24-30, 36-43) ${ }^{106}$ :

«Il leur proposa une autre parabole: "Il en va du Royaume des cieux comme d'un homme qui a semé du bon grain dans son champ. Pendant que les gens dormaient, son ennemi est venu; par-dessus, il a semé de l'ivraie en plein milieu du blé et il s'en est allé. Quand l'herbe eut poussé et produit l'épi, alors apparut aussi l'ivraie. Les serviteurs du maître de la maison vinrent lui dire : 'Seigneur, n'est-ce pas du bon grain que tu as semé dans ton champ ? D'où vient donc qu'il s'y trouve de l'ivraie ?' Il leur dit : 'C'est un ennemi qui a fait cela.' Les serviteurs lui disent: 'Alors, veux-tu que nous allions la ramasser ?' - 'Non, dit-il, de peur qu'en ramassant l'ivraie vous ne déraciniez le blé avec elle. Laissez l'un et l'autre croître ensemble jusqu'à la moisson, et au temps de la moisson je dirai aux moissonneurs : Ramassez d'abord l'ivraie et liez-la en bottes pour la brûler ; quant au blé, recueillez-le dans mon grenier.'"

"Alors, laissant les foules, il vint à la maison, et ses disciples s'approchèrent de lui et dirent: 'Explique-nous la parabole de l'ivraie dans le champ'. Il leur répondit: 'Celui qui sème le bon grain, c'est le Fils de l'homme ; le champ, c'est le monde ; le bon grain, ce sont les sujets du Royaume; l'ivraie, ce sont les sujets du Malin ; l'ennemi qui l'a semée, c'est le diable ; la moisson, c'est la fin du monde ; les moissonneurs, ce sont les anges. De même que

105. Erik Hornung, Der Eine und die Vielen. Ägyptische Götterwelt, Darmstadt, 1973, p. 150-151.

106. C. Focant, D. Marguerat, op. cit., p. 78-81. 
l'on ramasse l'ivraie pour la brûler au feu, ainsi en sera-t-il à la fin du monde : le Fils de l'homme enverra ses anges; ils ramasseront, pour les mettre hors de son Royaume, toutes les causes de chute et tous ceux qui commettent l'iniquité, et ils les jetteront dans la fournaise de feu; là seront les pleurs et les grincements de dents. Alors les justes resplendiront comme le soleil dans le Royaume de leur Père. Entende qui a des oreilles !' " ».

Dans cet extrait, «l'ennemi » correspond au démon, de même que dans la psychostasie égyptienne il désigne les adversaires d'Osiris et de Rê, reconnus pour leurs actions démoniaques.

\section{Conclusion}

L'étude comparative de l'interaction des intervenants dans les psychostasies égyptienne et chrétienne permet de classer ceux qui interviennent au tribunal comme « anges / génies » ou « démons », d'évaluer les différences et les similitudes entre les protagonistes et d'éclairer leurs rapports avec le bien et le mal. Le jugement se déroule sous la présidence d'Osiris, de Rê, ou du Christ. À la différence de l'entourage proche du Christ qui joue un rôle « direct » d'intercesseur, la famille d'Osiris, à savoir Isis, Nephthys et les quatre enfants d'Horus, veille sur le dieu osirien et « indirectement» sur le défunt justifié et identifié au dieu des morts. Thot, Anubis, Horus et Maât, qui examinent le cœur du mort à l'aide de la balance, sont investis de tâches avoisinant celles des anges, mais se distinguent d'eux par leur pouvoir décisif. Les quarante-deux assesseurs, qui écoutent la déclaration d'innocence du défunt, mettent à l'épreuve sa connaissance «théorique » des normes de la justice. Par la suite, l'ennéade procède à l'examen « pragmatique » de son cœur, témoin de l'application vertueuse des préceptes de Maât. Ce collège divin, qui siège dans la Salle des deux Maât, est analogue aux saints agissant comme assesseurs dans le Jugement dernier. Meskhenet, Chaï et Renoutet personnifient la récompense du défunt, s'opposant ainsi à la dévoreuse qui exécute le châtiment. Les quarante-deux assesseurs et la dévoreuse, souvent considérés comme figures démoniaques en raison de leur aspect terrifiant, de leur double fonction protectrice $\mathrm{du}$ juste et répressive de ses ennemis, et de leurs dénominations menaçantes, s'avèrent donc être des bourreaux ou des génies 
exécuteurs de la justice qui éliminent les malfaiteurs. Les fonctions de ce collège divin et du monstre correspondent à celles des anges qui, au au terme du Jugement dernier, punissent le diable et ses alliés. Ces derniers correspondent à Seth et à ses acolytes qui sont les vrais démons dans la psychostasie égyptienne. Les deux conceptions du jugement conservent le principe du mal au monde, qu'il s'agisse de Seth, d'Apohis ou du diable. Au contraire des instigateurs égyptiens du mal, qui sont châtiés de façon cyclique, c'est définitivement que le démon est contenu dans le jugement chrétien.

hgaber00@gmail.com 